\title{
Miniature ultrasound ring array transducers for transcranial ultrasound neuromodulation of freely-moving small animals
}

Kim, Hyunggug ; Kim, Seongyeon; Sim, Nam Suk; Pasquinelli, Cristina; Thielscher, Axel; Lee, Jeong Ho; Lee, Hyunjoo J.

Published in:

Brain Stimulation

Link to article, DOI:

10.1016/j.brs.2018.11.007

Publication date:

2018

Document Version

Peer reviewed version

Link back to DTU Orbit

Citation (APA):

Kim, H., Kim, S., Sim, N. S., Pasquinelli, C., Thielscher, A., Lee, J. H., \& Lee, H. J. (2018). Miniature ultrasound ring array transducers for transcranial ultrasound neuromodulation of freely-moving small animals. Brain Stimulation, 12(2), 251-255. https://doi.org/10.1016/j.brs.2018.11.007

\section{General rights}

Copyright and moral rights for the publications made accessible in the public portal are retained by the authors and/or other copyright owners and it is a condition of accessing publications that users recognise and abide by the legal requirements associated with these rights.

- Users may download and print one copy of any publication from the public portal for the purpose of private study or research.

- You may not further distribute the material or use it for any profit-making activity or commercial gain

- You may freely distribute the URL identifying the publication in the public portal 


\section{Accepted Manuscript}

Miniature ultrasound ring array transducers for transcranial ultrasound neuromodulation of freely-moving small animals

Hyunggug Kim, Seongyeon Kim, Nam Suk Sim, Cristina Pasquinelli, Axel Thielscher, Jeong Ho Lee, Hyunjoo J. Lee

PII: S1935-861X(18)30373-5

DOI: https://doi.org/10.1016/j.brs.2018.11.007

Reference: $\quad$ BRS 1351

To appear in: Brain Stimulation

Received Date: 16 June 2018

Revised Date: 7 November 2018

Accepted Date: 8 November 2018

Please cite this article as: Kim H, Kim S, Sim NS, Pasquinelli C, Thielscher A, Lee JH, Lee HJ, Miniature ultrasound ring array transducers for transcranial ultrasound neuromodulation of freely-moving small animals, Brain Stimulation, https://doi.org/10.1016/j.brs.2018.11.007.

This is a PDF file of an unedited manuscript that has been accepted for publication. As a service to our customers we are providing this early version of the manuscript. The manuscript will undergo copyediting, typesetting, and review of the resulting proof before it is published in its final form. Please note that during the production process errors may be discovered which could affect the content, and all legal disclaimers that apply to the journal pertain. 


\section{Miniature ultrasound ring array transducers for transcranial ultrasound neuromodulation of freely-moving small animals}

Hyunggug Kim ${ }^{1}$, Seongyeon Kim ${ }^{1}$, Nam Suk Sim ${ }^{2}$, Cristina Pasquinelli ${ }^{3,4}$, Axel Thielscher ${ }^{3,4}$, Jeong Ho Lee ${ }^{2}$, and Hyunjoo J. Lee ,** $^{1 *}$

${ }^{1}$ School of Electrical Engineering, Korea Advanced Institute of Science and Technology, Daejeon 34141, Republic of Korea

${ }^{2}$ Graduate School of Medical Science and Engineering, Korea Advanced Institute of Science and Technology,

Daejeon 34141, Republic of Korea

${ }^{3}$ Danish Research Centre for Magnetic Resonance, Centre for Functional and Diagnostic Imaging and Research, Copenhagen University Hospital Hvidovre, DK-2650 Hvidovre, Denmark

${ }^{4}$ Department of Electrical Engineering, Technical University of Denmark, DK-2800 Kgs. Lyngby, Denmark

\section{*Corresponding author:}

Hyunjoo Jenny Lee, Ph.D.

Korea Advanced Institute of Science and Technology

Room 4220, E3-2, Daehakro-291, Yuseong-gu,

Daejeon 34141, Republic of Korea

Tel.: +82 423507436 , Fax.: +82423507636

E-mail: hyunjoo.lee@kaist.ac.kr

Keyword: LIPUS, ultrasound transducer, freely-moving, neuromodulation 


\section{ABSTRACT}

Background: Current transcranial ultrasound stimulation for small animal in vivo experiment is limited to acute stimulation under anesthesia in stereotaxic fixation due to bulky and heavy curved transducers.

Methods: We developed a miniaturized ultrasound ring array transducer which is capable of invoking motor responses through neuromodulation of freely-moving awake mice.

Results: The developed transducer is a 32-element, $183-\mathrm{kHz}$ ring array with a weight of $0.035 \mathrm{~g}$ (with PCB: $0.73 \mathrm{~g}$ ), a diameter of $8.1 \mathrm{~mm}$, a focal length of $2.3 \mathrm{~mm}$, and lateral resolution of $2.75 \mathrm{~mm}$. By developing an affixation scheme suitable for freely-moving animals, the transducer was successfully coupled to the mouse brain and induced motor responses in both affixed and awake states.

Conclusion: Ultrasound neuromodulation of a freely-moving animal is now possible using the developed lightweight and compact system to conduct a versatile set of in vivo experiments.

\section{INTRODUCTION}

Transcranial focused ultrasound stimulation (tFUS) is a promising modality because of its competitive advantages such as focusing capability (i.e., high spatial resolution), beam steering capability, and long-term safety [1]. However, previous works on small animals have been limited to acute stimulation under various anesthetic levels (light to deep) in stereotaxic fixation [2-10] due to bulky and heavy curved transducers (Outer diameter: $>25 \mathrm{~mm}$; height: $\sim 40 \mathrm{~mm}$ ) with maximum intensities much larger than the required intensity range. Moreover, no in vivo ultrasound neuromodulation has been demonstrated under awake, head-fixed preparation such as spherical treadmill [11] and flat-floored air-lifted platform [12] for small animals. For a large non-human primate (NHP), sonication was delivered on both awake and sedated animals for brain-blood barrier (BBB) opening and neuromodulation [13-15]. However, even for the larger animal, head-fixation was still required for the experiment. There exists only one study which reported neuromodulation of local field potential (LFP) using a miniature single-element transducer [16]. Since the same stimulation modality is preferred to translate the findings from pre-clinical to clinical trials, to observe 
therapeutic effects of ultrasound neuromodulation on various disease models, it is important to develop an ultrasound neuromodulation system that supports freely-moving experiments. Moreover, such miniaturized neuromodulation tools could also facilitate the investigation of the biological mechanism of ultrasound neuromodulation [3, 17-24]. Here, we propose a light-weight capacitive micromachined ultrasonic transducer (CMUT) ring array suitable for non-invasive brain stimulation for chronic experiments. We demonstrate the feasibility of neuromodulation using the proposed miniaturized transducer ring array in both acute and awake in vivo mice experiments. This system enables freely-moving animal behavior studies where the effects of ultrasound neuromodulation could be observed in real-time in both acute and chronic conditions. For example, both immediate and chronic effects of ultrasound modulation on epilepsy frequency, sleep patterns, and cognitive performance could be observed using the proposed system.

\section{MATERIALS AND METHODS}

We designed and fabricated a ring array with an outer diameter of $8.1 \mathrm{~mm}$ and an inner diameter of $5.2 \mathrm{~mm}$ to generate a focal point at approximately $2.3 \mathrm{~mm}$ from the device with an immersion resonant frequency of $183 \mathrm{kHz}$ (Figure 1A, B, S1). Ring array was chosen because of the following advantages: natural focus at the center (Figure S2), larger aperture while minimizing localized skull heating, and extra room in the middle for integration with other devices. The ring array is composed of 32 elements, and each element is composed of 12 circular resonating plates (or cells) connected in parallel $[24,25]$. The weights of the ring array and fully-packaged array with a custom-designed printed circuit board (PCB) were $0.035 \mathrm{~g}$ and $0.73 \mathrm{~g}$, respectively (Figure 1C). For further information, see Supplementary Methods.

\section{RESULTS}

\section{Beam profile of the miniature ring array}

A volumetric hydrophone scan of 5-mm wide and 10-mm long was performed with a 0.25 -mm step from 
the center of the surface of the ring array (Figure S3). The CMUT ring array was biased at 100 DC voltage superimposed with a 183-kHz, 39.6 AC voltage. Full-Width Half-Maximum (FWHM) (i.e., focus size) of $10.13 \mathrm{~mm}^{2}$ in the horizontal plane and $6.12 \mathrm{~mm}^{2}$ in the vertical plane with a focus length of $2.3 \mathrm{~mm}$ and a maximum intensity of $50 \mathrm{~mW} / \mathrm{cm}^{2}(27 \mathrm{kPa})$ were observed (Figure $\left.1 \mathrm{~F}\right)$. These measurement results were comparable to the simulated beam profile (COMSOL Multiphysics ${ }^{\circledR}$, Burlington, MA, USA) (Figure 1E). The intensity at the focal point increased as the AC voltage increased where a maximum intensity of 174 $\mathrm{mW} / \mathrm{cm}^{2}(\sim 52 \mathrm{kPa})$ was achieved at an AC peak-to-peak voltage of $90 \mathrm{~V}$ (Figure 1D). In addition, while impedance measurement in air showed a resonant frequency of $\sim 780 \mathrm{kHz}$, Fast Fourier transform (FFT) of the measured transient pressure showed a center frequency of $183 \mathrm{kHz}$ and a $3 \mathrm{~dB}$ bandwidth of $179 \mathrm{kHz}$ (fractional bandwidth of $\sim 98 \%$ ) (Figure S4, S5). Lastly, to ensure that our device does not cause a significant heating to activate neuronal activities, we measured potential temperature increase using an Agarose gel phantom and a thermocouple. We observed a temperature increase of approximately $0.1^{\circ} \mathrm{C}$ after $\sim 240 \mathrm{~s}$ of continuous sonication (Figure S6). Since the duration of sonication in our in vivo protocol is only $0.2 \mathrm{~s}$, the temperature effect should be negligible.

\section{MR compatibility of the miniature ring array}

Since the target stimulation area is difficult to determine, non-invasive ultrasound neuromodulation could be accompanied with functional magnetic resonance imaging (fMRI). Thus, it is important for the CMUT device and the package system to be MR conditional. Here, we assessed the influence of the device with and without packaging on MR image quality using a 3T clinical scanner and an agar-filled spherical phantom. The device alone did not cause measurable effects on the MR images (Figure 1H) when compared to the baseline measurement (Figure 1G). Nevertheless, the initial measurement of the packaged device revealed clear distortions of the static magnetic field of the scanner, which were caused by the connector mounted on the PCB (Figure 1C). Without the connector, at the power-up with DC bias voltage alone, the adverse effects of the presence of the device on the MR images has substantially decreased (Figure 1I). In addition, while the 
device alone did not induce RF noise above the thermic noise floor, the noise was clearly measurable once the device was connected to the RF amplifier and DC power source placed outside of the MR cabin. The issues with the RF noise can be readily resolved using a physical filter attached to the MR cabin for future applications [26].

\section{Acute in vivo neuromodulation of motor cortex}

To confirm the functionality of the ring array, we performed in vivo acute mouse experiment without craniotomy. The CMUT array was biased at DC bias voltage of $100 \mathrm{~V}$ and driven with AC peak-to-peak voltage of $80 \mathrm{~V}$ at $183 \mathrm{kHz}$. Each stimulation trial consisted of 40-pulses of $90 \%$ duty cycle at a pulse repeat frequency (PRF) of $200 \mathrm{~Hz}$, and each 4.5-ms long pulse consisted of 756 pulses of ultrasound (Figure 2A, S7). During this trial, the total ultrasound power that was delivered (i.e., pulse intensity integral (PII)), was 0.28 $\mathrm{mJ} / \mathrm{cm}^{2}$ and spatial-peak, temporal-average intensity $\left(\mathrm{I}_{\text {spta }}\right)$ was $55.4 \mathrm{~mW} / \mathrm{cm}^{2}$. The success rate of motor responses was measured at an increasing intensity by adjusting the $\mathrm{AC}$ voltage. At each intensity, approximately 25 stimulation trials were conducted over $4 \mathrm{~min}$ and the event of 'success' and 'fail' was determined based on a threshold (i.e., 3 times the EMG noise floor) (Figure 2B, C). For all four mice, an increase in the success rate was observed as the intensity $\left(\mathrm{I}_{\text {spta }}\right)$ increased (Figure 2D), which was comparable to the ones demonstrated in the literature where bulky ultrasound transducer array was used [3]. At an intensity of $34.1 \mathrm{~mW} / \mathrm{cm}^{2}$, the average success rate of four mice was over $70 \%$ (see Movie $\mathrm{S} 1$ ).

Three control experiments were also performed to ensure that the motor responses were not evoked due to any potential electrical leakage or buzzing sound. First, the success rate of four mice was measured when the device was biased with DC bias voltage of $100 \mathrm{~V}$ and AC voltage of $0 \mathrm{~V}$. The observed success rate was negligible (i.e., $0 \sim 7.14 \%$ ) and was significantly lower than the success rate when AC voltage of $80 \mathrm{~V}$ was applied with the paired t-test $\mathrm{P}$ value of 0.0044 (Figure 2E). The second control experiment was performed by applying $0 \mathrm{DC}$ bias voltage with an AC voltage of $80 \mathrm{~V}$ on one mouse. The success rate of $6.8 \%$ was observed which was much lower than the success rate when the device was fully driven (i.e., $100 \mathrm{~V}_{\mathrm{DC}}+80 \mathrm{~V}_{\mathrm{AC}}$ ). Thus, 
the evoked motor responses were not due to any potential electrical leakage between the device. The last sham experiment was conducted to evaluate artifacts due to the buzzing sound of the transducer [27, 28]. While using the identical in vivo procedure and experimental setup, the packaged device was placed between the metal slits upside down. When the device was flipped, the success rate was $3.44 \%$ which was significantly smaller than that observed during the normal stimulation. When there was no device attached to the system, we have observed the success rate due to the spontaneous movements as high as $10.7 \%$. Thus, the effect of potential artifacts due to the buzzing sound should be negligible.

\section{In vivo neuromodulation of motor cortex of awake animals}

For neuromodulation of freely-moving mice, compact interface and package are essential. Specifically, design and implementation of three components were required: head fixture, collimator, and electric rotary joint to provide the input voltage to the CMUT ring arrays (Figure S8). Using this system, we successfully demonstrated the transcranial ultrasound neuromodulation of a freely-moving mouse where a success rate of $100 \%$ over 10 trials was observed when the device was fully driven (see Movie S2). We observed that the transducer coupled to the head did not cause impairment in its ability to walk, feed, and groom (see Movie S3). The stimulation was conducted approximately after 3 hours from the surgery. Although no EMG signals were recorded due to limited surgery techniques, the stimulation was visually recorded. After 7 days of the implantation, when additional ultrasound coupling gel was applied, we visually observed successful modulation over 10 trials. Not only our proposed interface system permitted stimulation of freely-moving animals over 7 days, the device was replaceable and reusable after the experiments. Lastly, we observed no significant tissue damage such as vascular hemorrhage and neuronal necrosis compared to that of the control mice (Figure S9). This result suggests that CMUT ring array produced no significant microscopic damage to the mouse cortex, which is similar to the results of single-element bulky transducers [29]. 


\section{DISCUSSION}

By devising a miniature ultrasound transducer array, we have demonstrated the possibility of performing transcranial ultrasound neuromodulation during both acute and awake states. Although more efforts are required to investigate the mechanism of ultrasound neuromodulation (indirect or direct) [19, 20], the proposed system provides the same functionalities as that of commercial bulky transducers but with a new capability of enabling freely-moving experiments. Although the intensity of our device was relatively low compared to the previous works $\left(\sim 10 \mathrm{~W} / \mathrm{cm}^{2}\right)$, we demonstrated that the intensity was sufficient for neuromodulation of motor cortex without craniotomy. To achieve a higher intensity while maintaining the resonant frequency, a thicker silicon circular plate with a smaller radius could be used [30]. In the succeeding developments, there are several limitations that needs be overcome. First, we have only tested our system on wild-type mice. We plan to apply our system to different animal disease models to demonstrate biologically meaningful therapeutic effects. Second, the fabricated device was in the form of an array, and thus, when interfaced with beam-forming circuits, dynamic focusing could be achieved to target different locations within the brain without relocating the device. For beam-steering, the individual element of the ring array must be also interfaced with a separate circuitry using multichannel driving systems such as commercially systems (e.g., Verasonics ${ }^{\circledR}$ ), custom-designed ICs, or multi-channel RF amplifiers. However, because of the inherent beam shape of the ring array, we still achieved a narrow single-focus along the stimulation axis which contributed to the simplification of our setup. Thus, future work includes interfacing our device with beam-forming circuitries for dynamic focusing and beam-steering.

\section{CONFLICT OF INTEREST}

None declared.

\section{APPENDIX A. SUPPLEMENTAL DATA}


Supplementary data related to this article can be found at https://doi.org/XXX.

\section{ACKNOWLEDGEMENTS}

This research was supported by the Brain Research Program through the National Research Foundation of Korea (NRF) funded by the Ministry of Science and ICT (2016M3C7A1904343), by the Engineering Research Center of Excellence (ERC) Program supported by National Research Foundation (NRF), Korean Ministry of Science \& ICT (MSIT) (Grant No. NRF-2017R1A5A1014708), by Nano Open Innovation Lab Project through National NanoFab Center (NNFC) funded by the Ministry of Science and ICT (COI1807M002), and a PhD stipend by the Technical University of Denmark.

\section{REFERENCES}

[1] Bystritsky A, Korb AS, Douglas PK, Cohen MS, Melega WP, Mulgaonkar AP, et al. A review of low-intensity focused ultrasound pulsation. Brain stimulation 2011;4(3):125-36.

[2] Tufail Y, Yoshihiro A, Pati S, Li MM, Tyler WJ. Ultrasonic neuromodulation by brain stimulation with transcranial ultrasound. Nature protocols 2011;6(9):1453-70.

[3] King RL, Brown JR, Newsome WT, Pauly KB. Effective parameters for ultrasound-induced in vivo neurostimulation. Ultrasound in medicine \& biology 2013;39(2):312-31.

[4] King RL, Brown JR, Pauly KB. Localization of ultrasound-induced in vivo neurostimulation in the mouse model. Ultrasound in medicine \& biology 2014;40(7):1512-22.

[5] Lee W, Kim H, Jung Y, Song I-U, Chung YA, Yoo S-S. Image-guided transcranial focused ultrasound stimulates human primary somatosensory cortex. Scientific reports $2015 ; 5$.

[6] Kamimura HA, Wang S, Chen H, Wang Q, Aurup C, Acosta C, et al. Focused ultrasound neuromodulation of cortical and subcortical brain structures using $1.9 \mathrm{MHz}$. Medical physics 2016;43(10):5730-5. 
[7] Li G-F, Zhao H-X, Zhou H, Yan F, Wang J-Y, Xu C-X, et al. Improved anatomical specificity of non-invasive neuro-stimulation by high frequency (5 MHz) ultrasound. Scientific reports 2016;6:24738.

[8] Eguchi K, Shindo T, Ito K, Ogata T, Kurosawa R, Kagaya Y, et al. Whole-brain low-intensity pulsed ultrasound therapy markedly improves cognitive dysfunctions in mouse models of dementia-Crucial roles of endothelial nitric oxide synthase. Brain Stimulation 2018.

[9] Yoo S-S, Bystritsky A, Lee J-H, Zhang Y, Fischer K, Min B-K, et al. Focused ultrasound modulates region-specific brain activity. Neuroimage 2011;56(3):1267-75.

[10] Kim H, Taghados SJ, Fischer K, Maeng L-S, Park S, Yoo S-S. Noninvasive transcranial stimulation of rat abducens nerve by focused ultrasound. Ultrasound in medicine \& biology 2012;38(9):1568-75.

[11] Dombeck DA, Khabbaz AN, Collman F, Adelman TL, Tank DW. Imaging large-scale neural activity with cellular resolution in awake, mobile mice. Neuron 2007;56(1):43-57.

[12] Kislin M, Mugantseva E, Molotkov D, Kulesskaya N, Khirug S, Kirilkin I, et al. Flat-floored air-lifted platform: a new method for combining behavior with microscopy or electrophysiology on awake freely moving rodents. Journal of visualized experiments: JoVE 2014(88).

[13] Deffieux T, Younan Y, Wattiez N, Tanter M, Pouget P, Aubry J-F. Low-intensity focused ultrasound modulates monkey visuomotor behavior. Current Biology 2013;23(23):2430-3.

[14] Yang P-F, Phipps MA, Newton AT, Chaplin V, Gore JC, Caskey CF, et al. Neuromodulation of sensory networks in monkey brain by focused ultrasound with MRI guidance and detection. Scientific reports 2018;8(1):7993.

[15] Downs ME, Buch A, Karakatsani ME, Konofagou EE, Ferrera VP. Blood-brain barrier opening in behaving non-human primates via focused ultrasound with systemically administered microbubbles. Scientific reports 2015;5:15076.

[16] Li G, Qiu W, Zhang Z, Jiang Q, Su M, Cai R, et al. Noninvasive Ultrasonic Neuromodulation in Freely Moving Mice. IEEE Transactions on Biomedical Engineering 2018. 
[17] Tyler WJ. Noninvasive neuromodulation with ultrasound? A continuum mechanics hypothesis. The Neuroscientist 2011;17(1):25-36.

[18] Kubanek J, Shi J, Marsh J, Chen D, Deng C, Cui J. Ultrasound modulates ion channel currents. Scientific reports 2016;6.

[19] Guo H, Hamilton M, Offutt SJ, Gloeckner CD, Li T, Kim Y, et al. Ultrasound produces extensive brain activation via a cochlear pathway. Neuron 2018.

[20] Sato T, Shapiro MG, Tsao DY. Ultrasonic neuromodulation causes widespread cortical activation via an indirect auditory mechanism. Neuron 2018.

[21] Shin H, Lee HJ, Chae U, Kim H, Kim J, Choi N, et al. Neural probes with multi-drug delivery capability. Lab on a Chip 2015;15(18):3730-7.

[22] Lee HJ, Choi N, Yoon E-S, Cho I-J. MEMS devices for drug delivery. Advanced drug delivery reviews 2018;128:132-47.

[23] Lee HJ, Son Y, Kim D, Kim YK, Choi N, Yoon E-S, et al. A new thin silicon microneedle with an embedded microchannel for deep brain drug infusion. Sensors and Actuators B: Chemical 2015;209:413-22. [24] Park S, Yoon I, Lee S, Kim H, Seo J-W, Chung Y, et al. CMUT-based resonant gas sensor array for VOC detection with low operating voltage. Sensors and Actuators B: Chemical 2018;273:1556-63.

[25] Park KK, Lee HJ, Kupnik M, Khuri-Yakub BT. Fabrication of capacitive micromachined ultrasonic transducers via local oxidation and direct wafer bonding. Journal of microelectromechanical Systems 2011;20(1):95-103.

[26] Moisa M, Pohmann R, Ewald L, Thielscher A. New coil positioning method for interleaved transcranial magnetic stimulation (TMS)/functional MRI (fMRI) and its validation in a motor cortex study. Journal of Magnetic Resonance Imaging 2009;29(1):189-97.

[27] Legon W, Bansal P, Tyshynsky R, Ai L, Mueller JK. Transcranial focused ultrasound neuromodulation of the human primary motor cortex. bioRxiv 2018:234666. 
[28] Mueller J, Legon W, Opitz A, Sato TF, Tyler WJ. Transcranial focused ultrasound modulates intrinsic and evoked EEG dynamics. Brain stimulation 2014;7(6):900-8.

[29] Tufail Y, Matyushov A, Baldwin N, Tauchmann ML, Georges J, Yoshihiro A, et al. Transcranial pulsed ultrasound stimulates intact brain circuits. Neuron 2010;66(5):681-94.

[30] Wygant IO, Kupnik M, Khuri-Yakub BT, Wochner MS, Wright WM, Hamilton MF. The design and characterization of capacitive micromachined ultrasonic transducers (CMUTs) for generating high-intensity ultrasound for transmission of directional audio. Ultrasonics Symposium, 2008. IUS 2008. IEEE. IEEE; 2008:2100-2.

\section{FIGURE LEGENDS}

Figure 1 | Miniature transducer for transcranial ultrasound stimulation of freely-moving animals. (A) Conceptual 3D schematics of proposed ring array composed of 32 elements and a single element composed of 12 circular cells. (B) Photograph of the fabricated 32-element CMUT ring array. (C) Photograph of the CMUT ring array wire-bonded and packaged on a custom-designed PCB with a connector. (D) Measured intensity at the focal point over different $A C$ voltages $\left(V_{A C}\right)$. (E) Simulated 2D beam profile in the axial and radial directions. (F) Measured 2D beam profile in the axial and radial directions. XY plans were obtained at focal depth (z-axis = $2.3 \mathrm{~mm})(\mathbf{G})$ EPI image (top) and field map (bottom) of the baseline measurements without the device. Shown is a horizontal slice through the center of the phantom. (H) EPI image (top) and field map (bottom) of the CMUT ring array. The device was placed centrally on the top of the phantom. The EPI image did not show any signs of distortion or signal dropout. The field map revealed B0 inhomogeneities that are in the same range as seen in the baseline measurements. (I) EPI image (top) and field map (bottom) of the powered-up device connected to the bias tee via copper wires directly soldered to the PCB. Both the EPI image and the field map demonstrated the MR conditionality of the packaged device. 
Figure 2. Ultrasound neuromodulation of motor cortex using CMUT ring array. (A) Schematics of the trigger waveform delivering 40 pulses of 756 ultrasound pulses. The total power delivered through this waveform is described in different metrics. (B) Schematics illustrating 'success' events determined when the EMG power was larger than 3 times the noise floor. (C) Example of recorded raw EMG signals at the incident of (i) 'fail' and (ii) 'success' events. (D) Success rate measured over approximately 25 stimulation trials for 4 mice at varying ultrasound intensities (ISPPA) controlled by AC voltages. (E) Success rate of a control case when $100 \mathrm{DC}$ voltage $\left(\mathrm{V}_{\mathrm{DC}}\right)$ was applied with $0 \mathrm{~V}_{\mathrm{AC}}$. 
A

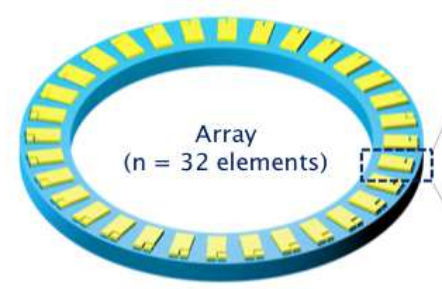

C

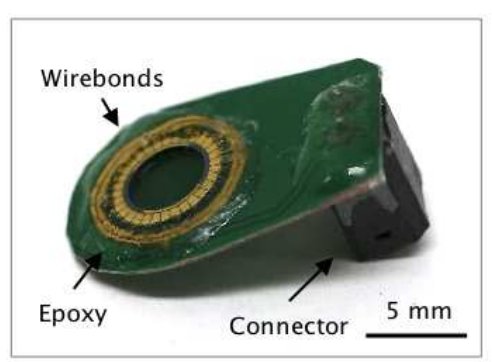

D

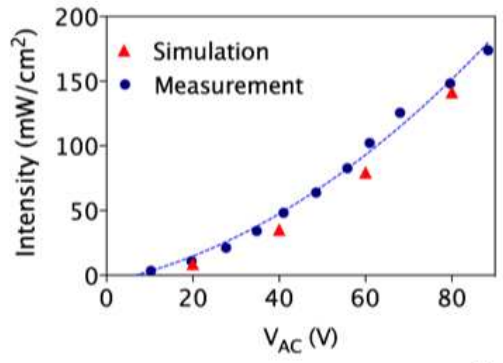

G

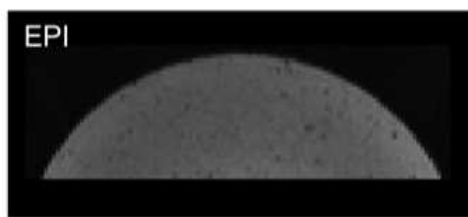

Field map

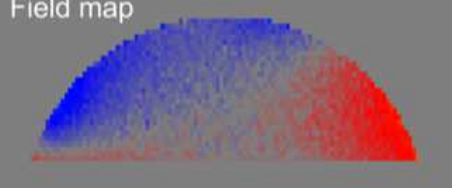

Single element ( $m=12$ cells)

$$
\leftarrow \begin{gathered}
\text { Ground } \\
\text { pad }
\end{gathered}
$$

E

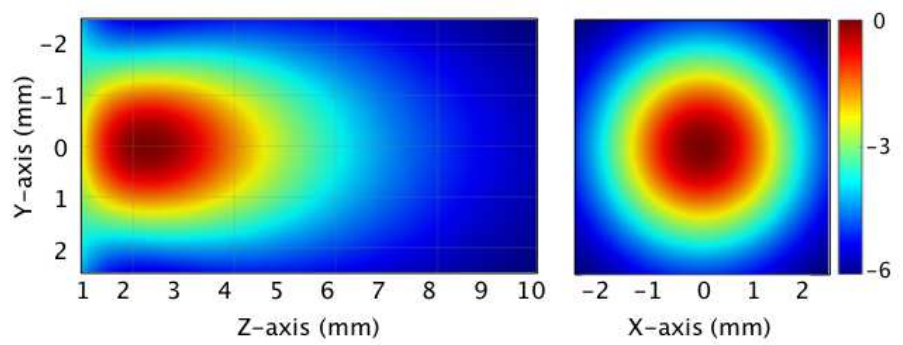

F

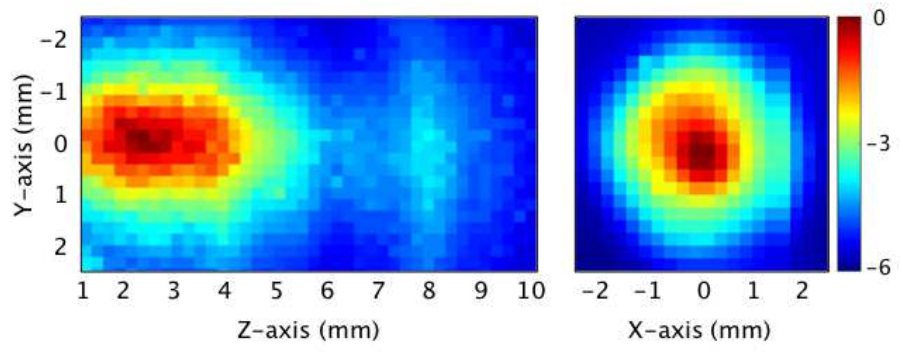

I

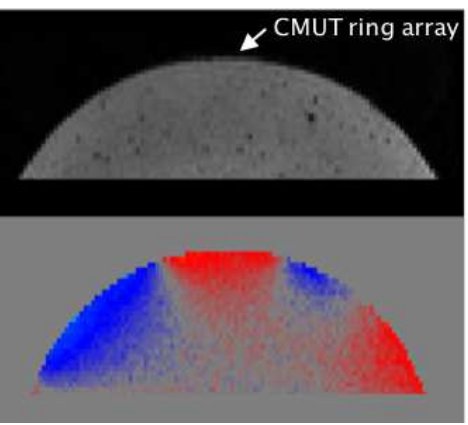

B

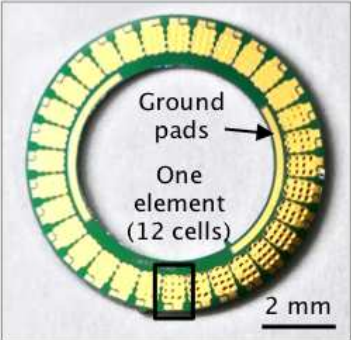

$\mathrm{mm}$

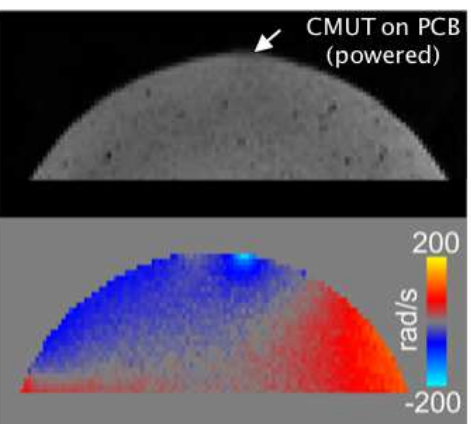


A

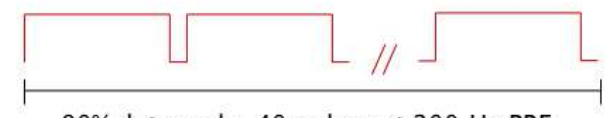

$90 \%$ duty cycle, 40 pulses at $200 \mathrm{~Hz}$ PRF

Total stimulation time: $200 \mathrm{~ms}$

ㄱ|

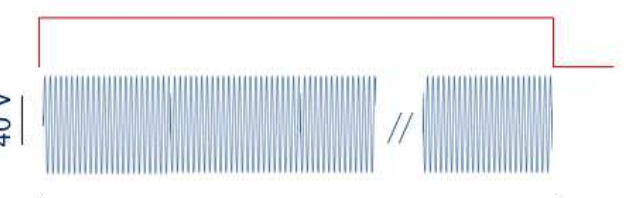

756 pulses of $f_{0}=168 \mathrm{kHz}$ over $4.5 \mathrm{~ms}$

C

(i) EMC signals (Fail)

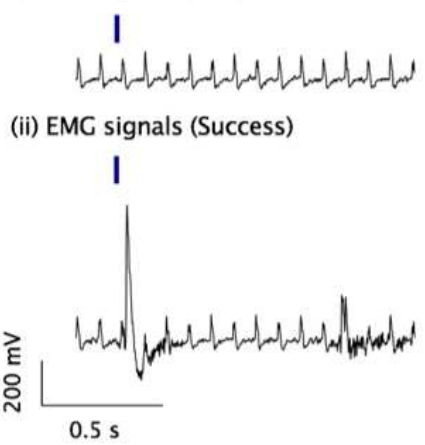

US Power

For $\mathrm{V}_{\mathrm{AC}}=80 \mathrm{~V}$, $P_{\max }=52 \mathrm{kPa}$

$P I I=0.238 \mathrm{~mJ} / \mathrm{cm}^{2}$

$I_{\text {SPPA }}=530 \mathrm{~mW} / \mathrm{cm}^{2}$

$I_{\text {SPTA }}=47.4 \mathrm{~mW} / \mathrm{cm}^{2}$

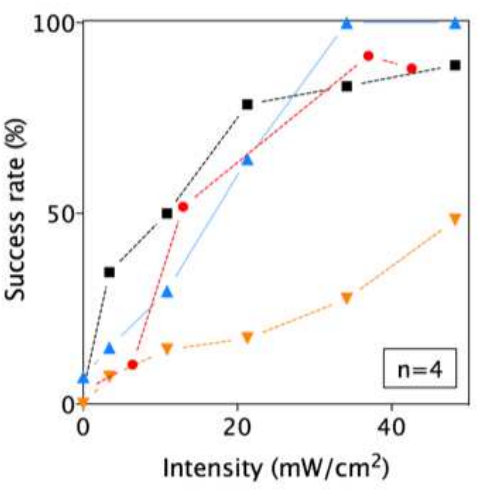

B

(i) Ultrasound trigger

l I I I

(ii) Success event

I

I

(iii) EMG signals

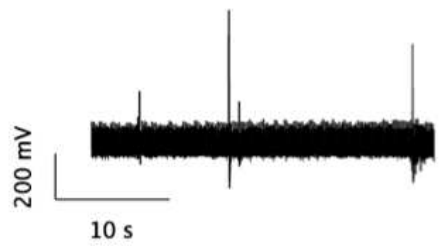

E

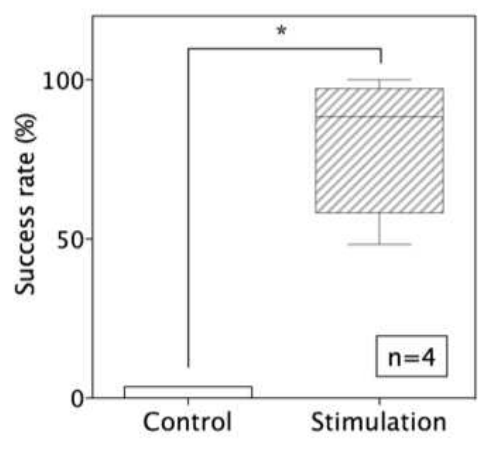

